Commentary/Todd \& Gigerenzer: Simple heuristics

\title{
On the descriptive validity and prescriptive utility of fast and frugal models
}

\author{
Clare Harries ${ }^{1}$ and Mandeep K. Dhami ${ }^{2}$ \\ ${ }^{1}$ Department of Psychology, University College London, London WC1E6BT, \\ United Kingdom; ${ }^{2}$ Department of Psychology, City University, London EC1V \\ OHB, United Kingdom. clare.harries@ucl.ac.uk \\ www.psychol.ucl.ac.uk/clare/self.html m.k.dhami@city.ac.uk
}

Abstract: Simple heuristics and regression models make different assumptions about behaviour. Both the environment and judgment can be described as fast and frugal. We do not know whether humans are successful when being fast and frugal. We must assess both global accuracy and the costs of Type I and II errors. These may be "smart heuristics that make researchers look simple."

Are humans really fast and frugal? Should humans be fast and frugal? Human judgment may be described on a number of dimensions such as the amount of information used and how it is integrated. The choice of a model dictates how these dimensions are characterised, irrespective of the data. For example, regression models in judgment and decision-making research are linear and compensatory, and researchers have assumed that humans are, too (Cooksey 1996). The fast and frugal models proposed by Gigerenzer, Todd, and the ABC Research Group (1999) use few cues and are often noncompensatory, assuming humans are, too.

Are humans really using less information in the fast and frugal model than the regression model? People can chunk information (e.g., Simon 1979). Regression models are characterised as complex in terms of use of multiple cues, but they often contain few significant cues (on average three; Brehmer 1994). This challenges the argument that fast and frugal models are more frugal than regression models, at least in terms of the number of cues searched. Unlike standard practice (Tabachnik \& Fidell 1996), in their regression analyses, Gigerenzer et al. retain nonsignificant cue weights. A fairer test would compare fast and frugal models against parsimonious regression models.

Regression models have been used to describe the relationship between judgments and the cues (the judgment system), and the relationship between outcomes and the cues (the environment system; Cooksey 1996). In both cases the underlying structure of the cues is similar (e.g., they are correlated). Fifteen years of machine learning research demonstrates that fast and frugal models can describe environments (Dutton \& Conroy 1996). It is not surprising that these models should also be good at describing human 
judgment. Both types of models are useful for fitting data per se. Chapter 7 demonstrates that fast and frugal models are good at describing human judgment, under conditions of time pressure with participants neither experienced nor familiar with the choice task. At other points in the book, results of simulations seem to be generalised with little justification to humans. However, research has shown that fast and frugal models are valid descriptions of professionals' judgment behaviour in the legal and medical domains (Dhami \& Ayton 1998; Harries \& Dhami 1998). So far we have learned that the environment and humans can be described as fast and frugal. We do not yet know whether humans are successful when being fast and frugal because studies either don't collect judgments or don't include outcomes.

We have doubts about the prescriptive utility of fast and frugal models. The book argues (at least implicitly) that if fast and frugal models are good at predicting the environment, then humans should (and do) use these strategies to make accurate decisions. However, in many situations global accuracy is not the first concern: the two types of errors (Type I and Type II) are differentially weighted. In medicine, for example, all tests involve a trade off between Type I and Type II errors. Researchers developing machine-learning models in this domain incorporate the costs of the two types of error (Kukar et al., 1999). In criminal justice, the opposing concepts of due process and of crime control attempt to reduce crime whilst minimising different types of error (Type Ithe number of people falsely convicted and Type II-the number of guilty people acquitted, respectively). It so happens that due process expects a regression-like behaviour: all available information is searched, weighted, and integrated. The fast and frugal strategy of crime control emphasises information associated with guilt, encouraging "conveyor belt justice." Justice is synonymous with due process, so the judge should behave like a regression model. Of course, unlike medicine, the socially constructed nature of criminal justice implies that we could change our notion of justice to one that reflects fast and frugal behaviour. Before Gigerenzer et al. encourage this we recommend that they evaluate models on other criteria in addition to global accuracy. In sum, the descriptive success of simple heuristics does not, of itself, imply their prescriptive utility.

On a differing point, if we do find that fast and frugal models have prescriptive utility, their potential as cognitive aids or in cognitive feedback vastly outweighs that of regression models (which have done pretty well; Cooksey 1996). Fast and frugal models are easy to understand and to apply while regression models are difficult to use without an aid and without knowing the range of cases on which they were formed.

In short, we welcome fast and frugal models because they make us re-think the dimensions of human judgment. The danger is that they will be automatically adopted as tools to describe human judgment, like regression models have been for 50 years. All models are paramorphic not isomorphic. So, we should be wary of "smart heuristics that make researchers look simple." 